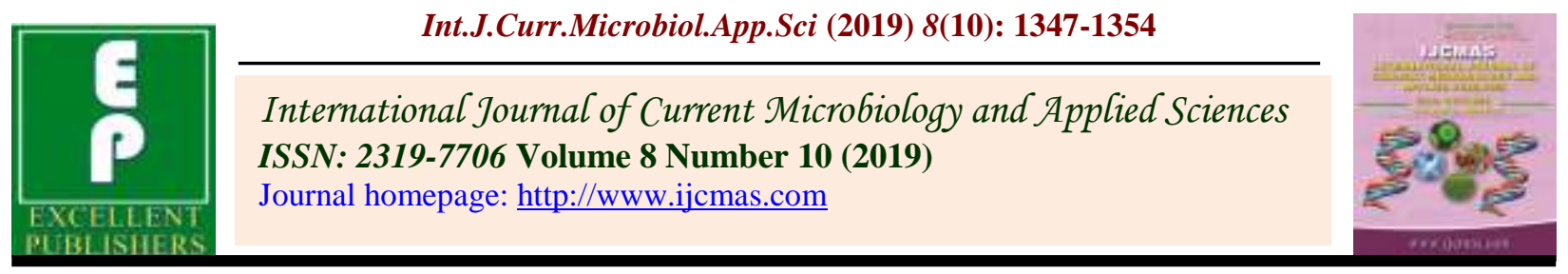

Original Research Article

https://doi.org/10.20546/ijcmas.2019.810.157

\title{
Mastitis Prevalence in Villages of District Sonipat and Panipat, Haryana
}

\author{
Samanwita Banerjee*, Anil Kanaujia, Rajkumar Yadav and Mohan JI Saxena \\ Research and Development Centre, Ayurvet Research Foundation, Village, \\ Chidana, Sonepat-131306, Haryana, India
}

*Corresponding author

\section{A B S T R A C T}

Keywords

Mastitis,

Occurrence,

Pathogens, $S$.

aureus, E. coli,

Antibiotic

sensitivity test

\section{Article Info}

Accepted:

12 September 2019

Available Online:

10 October 2019
The outbreak of mastitis lead to heavy economic losses in dairy industries by deteriorating the quality and quantity of milk. Inappropriate usage of antimicrobials such as wrong dose, drug or duration may contribute to the increase in antimicrobial resistance (AMR) without improving the outcome of treatment. Mastilep, a non-antibiotic polyherbal gel containing herbal ingredients is generally applied on udder and teats as precautionary measure and for treatment of subclinical mastitis and clinical mastitis as a co therapeutic medicine along with the prescribed doses of antibiotics. A study at ARF R\&D Centre was taken up for determination of the mastitis causing organisms in dairy animals and its sensitivity towards commonly used antibiotics. Overall, it was found that Ceftriaxone/tazobactam, Ciprofloxacin, Cefoperozone/sulbactam, Oxytetracycline, Neomycin, Chloramphenicol were more sensitive than rest of the antibiotics. General awareness among dairy farmers about hygiene, proper milking procedure is need of hour. Proper clinical investigation of cases and appropriate antibiotic and non antibiotic based polyherbal medication will definitely help in alleviating antimicrobial resistance menace.

\section{Introduction}

Mastitis is the most dreaded disease for dairy farmers owing to reduced milk production, increased treatment costs, labor, milk discarding following treatment, death and premature culling. Over 135 organisms have been documented as causative pathogens for bovine mastitis. Hygiene, environmental' factors and metabolic disturbances derived from high milk yield combine to create conditions favorable to the onset of mastitis which occurs in both clinical and subclinical forms (Rathi et al., 2008). Clinical form of the disease is an individual animal problem and can be easily diagnosed during clinical examination whereas, subclinical mastitis is a herd problem which may go unnoticed since no gross signs of inflammation and changes in the milk composition are evident and the milk 
and udder appear normal (Rakesh et al., 2018). Symptoms for the disease include inflammation of the mammary glands along with other mild signs of flakes or clots in the milk and the animal may have slight swelling of infected quarter, fever, rapid pulse, loss of appetite, dehydration, elevated somatic cell count (SCC) of the milk (Rathi et al., 2008).

Control of bovine mastitis is constrained because of multiple etiological agents, lack of awareness among farmers of the subclinical form of the disease, and this aspect is of fundamental importance because of the possibility of spreading infectious agents through the herd, public health risk due to consumption of unsafe milk, and less efficient processing of milk (Krishnakumar et al., 2017). Inappropriate usage of antimicrobials such as wrong dose, drug or duration may contribute to the increase in antimicrobial resistance without improving the outcome of treatment (Singh et al., 2018). Mastilep, a proprietary polyherbal formulation of AYURVET for dairy animals is a nonantibiotic polyherbal gel containing herbal ingredients such as Glycyrrhiza glabra, Curcuma longa, Cedrus deodara, Eucalyptus globulus, etc. is usually applied on udder and teats as precautionary measure and for treatment of subclinical mastitis, whereas in clinical mastitis, it is applied on the affected teat and udder area twice in a day after milking for 7 days as a co therapeutic medicine along with the prescribed doses of antibiotics.

Haryana is known for milk production, Sahiwal cows and the world's best buffalo the Murrah capable of milk yields as high as 35 $\mathrm{kg}$ a day. A study at ARF R\&D Centre was taken up for determination of the mastitis causal organisms in traditionally managed dairy animal and its sensitivity towards commonly used antibiotics in districts of Panipat and Sonipat.

\section{Materials and Methods}

\section{Sample size and sampling method}

100 milk samples were collected from different villages of district Sonipat and Panipat. Prior to collection, few questionnaires were filled up to the milking procedure, previous history of mastitis, quantity and quality of milk, duration of illness, symptoms (if any) etc.

The sampling was done after treating the teat end with $\mathrm{KMnO}_{4}$ and allowed to dry. First few streams of milk were discarded. The samples were collected aseptically in a sterile tube and transferred to the microbiology lab for analysis under cold condition

\section{pH test}

The $\mathrm{pH}$ of the milk samples was checked on previously calibrated digital $\mathrm{pH}$ meter.

\section{Sodium Lauryl Sulfate Test (SLST)}

Prepared 3\% Sodium Lauryl Sulfate solution $(\mathrm{pH}=10.3)$ and added bromothymol blue as an indicator. Mixed same proportion of milk sample and 3\% Sodium Lauryl Sulfate solution in a clean petri dish and swirled for 1 minutes, leading to ruptured of the somatic cell and release DNA. Being acidic in nature DNA binds with akly-arylsulfonate in solution and lead to the formation of gel (Dharamsheela Thakur et al., 2018). The consistency of gel grades the severity of mastitis from severe to low risk.

\section{Antibiotic susceptibility testing of bacterial isolates}

\section{Isolation of bacteria}

Inoculated $10 \mathrm{ml}$ of sample into $90 \mathrm{ml}$ of sterile nutrient broth and incubated at $37^{\circ} \mathrm{C}$ for 24 
hours. After incubation of $24 \mathrm{hrs}$, streaked onto nutrient agar, differential media and selective media and incubated at $37^{\circ} \mathrm{C}$ for 24 hours. The characteristic colonies were observed on respective media and gram staining was done.

\section{Identification of bacteria}

The primary identification was made with colony characteristic, morphology, pigmentation, hemolytic characteristic followed by gram staining. Biochemical tests were done accordingly for identification of different bacterial isolates ((A. Singh et al., 2018), (Barrow and Feltham, 1993).

\section{Culture sensitivity test}

18 commonly used Antibiotics by Veterinarian in the district of Sonipat and Panipat were studied against bacterial isolates. Inoculated $10 \mathrm{ml}$ of sample into $90 \mathrm{ml}$ of sterile nutrient broth and incubated at $37^{\circ} \mathrm{C}$ for 24 hours. After enrichment, smeared onto to Mueller Hinton agar with cotton swab uniformly and kept the plates at room temperature for $30 \mathrm{mins}$ to allow the inoculum to be absorbed on the media. After short incubation, antibiotic discs were placed onto the plate at equal distance for proper zone of inhibition and incubate the plate at $37^{\circ} \mathrm{C}$ for 18- 24hrs shown in Figure 1.

The zone of inhibition on the plate was measured in millimeter $(\mathrm{mm})$ by Vernier caliper and was compared with standard chart of zone of inhibition supplied by the manufacturer (Bauer, A.W et al., 1966). Antibiotics used were Amoxicillin/sulbactam, Ampicillin, Amoxyclav, Ceftazidime, Ceftizoxime, Ceftriaxone, Chloramphenicol, Enrofloxacin, Gentamicin, Neomycin, Cefoperozone/sulbactam, Streptomycin, Oxytetracycline, Ceftriaxone/tazobactam, Ampicillin/Cloxacillin and Kanamycin.

\section{Results and Discussion}

Out of 100 samples, 64 samples (64\%) were from Sonipat district whereas 36 samples $(36 \%)$ from Panipat. In the study, in Sonipat district we found that $79.69 \%$ cases were clinical mastitis whereas $20.31 \%$ samples were subclinical. Similarly, in Panipat, $94.44 \%$ were clinical and $5.56 \%$ were subclinical [Fig. 2]. The clinical samples were associated with redness, swollen and hardness of udder, discoloration of milk such as yellowish milk, reddish milk with precipitates, flakes, clots, exudate, etc. In few cases, it was observed that quantity of milk was reduced and co-existed with physical discomfort such nausea, fever, dullness, and loss of appetite.

Subclinical samples were confirmed by increased in $\mathrm{pH}$ up to 8.0 and light to dense clotting in SLST.

Predominant mastitis causing microorganisms were Staphylococcus aureus (35.56\%) followed by Escherichia coli (8.89\%), Streptococcus spp. (4.44\%) Enterobacter spp. (3.33\%), Enterococcus. sp. (2.22\%), Bacillus spp. (2.22\%) and Diplococci sp. (2.22\%) Pseudomonas aeruginosa (1.11\%). Bacillus sp. is considered to be non-importance causal organism for mastitis because it has been found in environment such as air, water soil, etc. This showed that the hygienic condition of the environment or milking procedure is poor which lead to mastitis in bovine.

Apart from that $40.23 \%$ of total cases are due to the mixed infection i.e. the combination of two or more microorganisms shown in Figure 3a. Maximum cases were found due to the combination of Staphylococcus aureus and Escherichia coli $(72.22 \%)$ followed by other combination shown in Figure $3 b$.

During the study, 8 isolates showed different percentage of susceptibility against 18 
different antibiotics. Among 18 different antibiotics, isolates showed highest sensitivity against Ceftriaxone/tazobactam (CIT) $76.25 \%$ followed by Ciprofloxacin (71.85\%), Cefoperozone/ Sulbactam (68.49\%), Oxytetracycline (62.41\%), Neomycin (54.09\%), Chloramphenicol (51.08\%), Gentamycin (47.46\%), Ceftriaxone (44.12\%), Kanamycin (43.81\%), Streptomycin (43.04\%), Enrofloxacin (41.62\%), Ceftizoxime (38.62\%), Ceftazidime (36.75\%), Ampicillin (17.88\%), Amoxicillin/Sulbactam (13.65\%), Cefotaxime (7.62\%), Ampicillin/Cloxacillin (6.80\%) and Amoxiclav (6.76\%). The overall susceptibility of antibiotic against isolates is shown in Figure 4.

Antibiogram profile using 18 antibiotics revealed wide range of reactivity against different isolates. Staphylococcus aureus were highly sensitive to Ceftriaxone / tazobactam (73.91\%), Neomycin (69.57\%), Ciprofloxacin (63.64\%), Chloramphenicol (62.50\%), Cefoperozone / sulbactam (60.87\%), whereas Escherichia coli were highly sensitive to Neomycin $(100 \%)$ followed by Chloramphenicol (75\%), Oxytetracycline (75\%), Enrofloxacin (62.50\%), Ciprofloxacin (50\%), Cefoperozone / sulbactam (50\%), and rest of the antibiotics are less than $50 \%$ shown in Figure.5a. Streptococcus spp. were highly sensitive to Neomycin (100\%), Ceftriaxone / tazobactam (100\%), Ceftriaxone (100\%) followed by Ciprofloxacin (75\%), Ceftizoxime (66.67\%), Ceftazidime (50\%), Chloramphenicol (50\%), Oxytetracycline (50\%), Kanamycin (50\%), Gentamicin $(50 \%)$ whereas Bacillus spp. showed sensitivity to Chloramphenicol (100\%), Ceftriaxone / tazobactam (100\%), Oxytetracycline (100\%), Kanamycin (100\%), Enrofloxacin (50\%), Ciprofloxacin (50\%), Cefoperozone / sulbactam (50\%), Streptomycin (50\%) as shown in Figure 5a. Diplococci. sp. was only sensitive to Ampicillin (50\%), Enrofloxacin
(50\%), Cefotaxime (50\%), Ciprofloxacin $(50 \%)$, Gentamicin $(50 \%)$ and were resistant to rest aforementioned antibiotics.

Enterobacter spp. showed $100 \%$ sensitivity to Ciprofloxacin, Gentamycin, Neomycin, Cefoperozone / sulbactam, Ceftriaxone / tazobactam, Oxytetracycline, Kanamycin followed by Enrofloxacin (66.67\%), Streptomycin $(66.67 \%)$, as shown in Figure $5 b$.

Enterococcus spp. and Pseudomonas aeruginosa showed consistency in sensitivity to Ceftazidime (100\%), Ceftizoxime (100\%), Ciprofloxacin (100\%) and Cefoperozone / sulbactam (100\%). Enterococcus spp. showed $50 \%$ sensitivity to Ampicillin, Amoxicillin /sulbactam, Ceftriaxone, Chloramphenicol, Enrofloxacin, Streptomycin, Oxytetracycline and Ceftriaxone / tazobactam whereas Pseudomonas aeruginosa showed $100 \%$ to Ceftriaxone, Ceftriaxone / tazobactam and Streptomycin as shown in Figure 5b.

In mixed infection, it was found that $88.89 \%$ of the infections were sensitive to Chloramphenicol followed by Oxytetracycline (76.47\%), Gentamicin (71.88\%), Neomycin (63.16\%), Ceftriaxone / tazobactam (61.11\%), Ciprofloxacin (58.06\%) and Cefoperozone / sulbactam $(55.56 \%)$. Rest all the antibiotics were minimal sensitive against mixed infection as shown in Figure 5b.

The present study reveals that clinical cases are higher in number than the sub-clinical cases in all the aforementioned districts of Haryana. It was also found that the mixed infections cases are more than the single isolate infection. Among mixed infection, maximum cases were due to the infection of $S$. aureus and E. coli which is major source of fecal contamination. 
Picture.1 Udder infected with mastitis

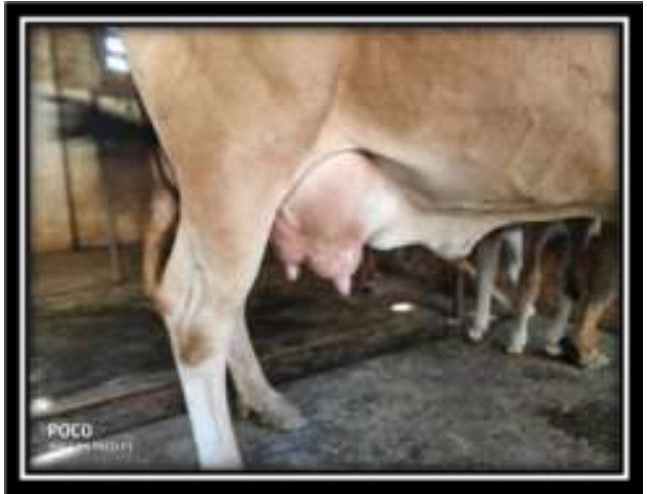

Fig.1 Microbiological zone of inhibition

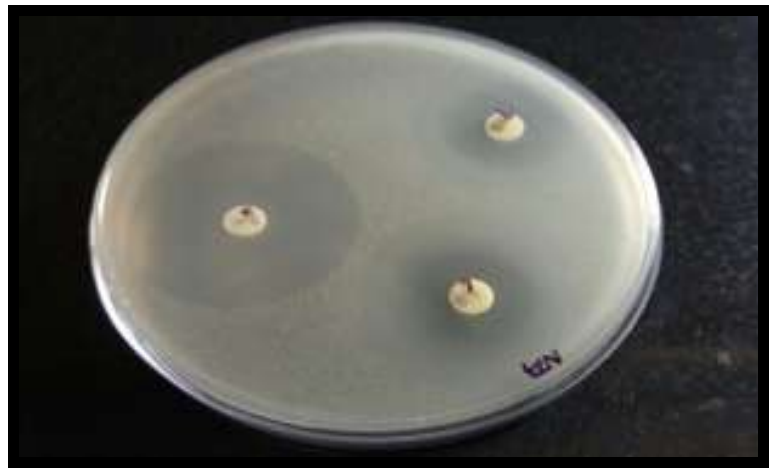

Fig.2 Percentage of different mastitis from different districts

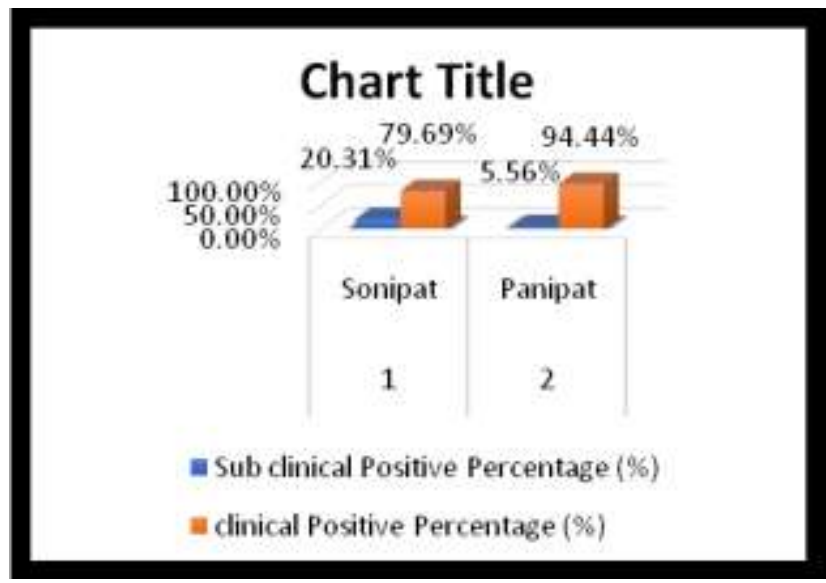


Fig.3a Percentage of causal Microorganism

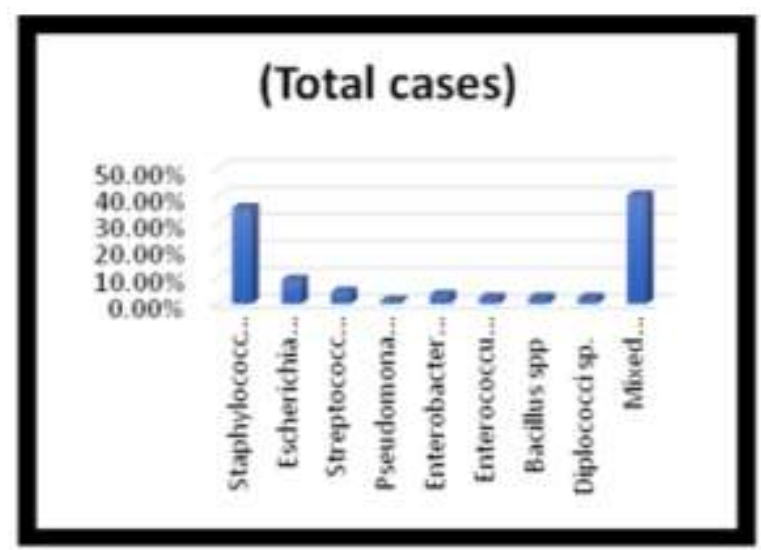

Staphylococcus aureus-(35.56\%), Escherichia coli-(8.89\%), Streptococcus sp.-(4.44\%), Pseudomonas aeruginosa-(1.11\%), Enterobacter sp.-(3.33\%), Enterococcus. Sp.-(2.22\%), Bacillus sp.- (2.22\%), Diplococci sp.-(2.22\%), Mixed infection-(40.23\%).

Fig.3b Microorganism associated with mixed infection

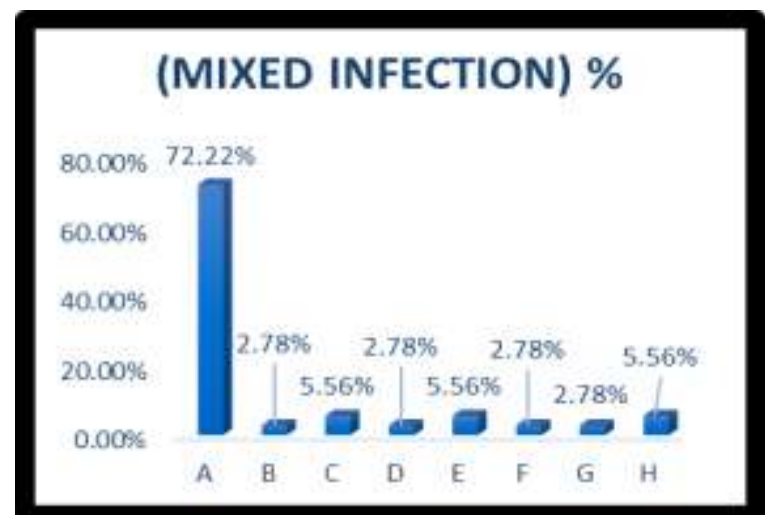

(A)- S. aureus, E. coli, (B)- E. coli, candida albican, (C)- S. aureus, Diplococci.sp, (D)- S. aureus, E. coli, diplococci, (E)- S. aureus Streptococcus.spp, (F)- S. aureus, E. coli, Streptococcus. Spp., (G)- S. aureus, P. aeruginosa, (H)- S. aureus, Rhodotorula

Fig.4 Reactivity\% of different antibiotics

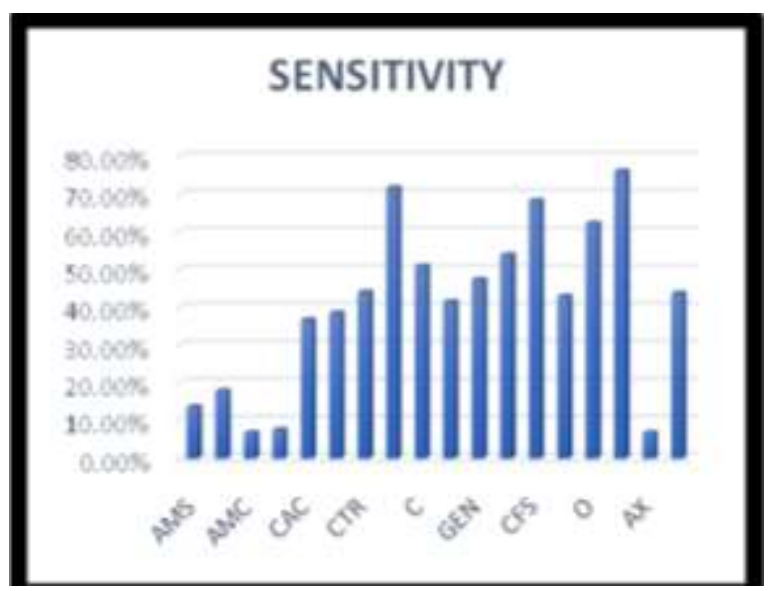

Abbreviate: Amoxicillin/sulbactam - (AMS), Ampicillin - (AMP), Amoxyclav - (AMC), Cefotaxime - (CTX), Ceftazidime (CAC), Ceftizoxime - (CZX), Ceftriaxone - (CTR), Ciprofloxacin - (CIP), Chloramphenicol (C), Enrofloxacin (EX), Gentamicin (GEN), Neomycin (N), Cefoperozone/ sulbactam - (CFS), Streptomycin - (S), Oxytetracycline - (O), Ceftriaxone tazobactam (CIT), Ampicillin/Cloxacillin - (AX), Kanamycin - (K). 
Fig.5a Sensitivity of Staphylococcus aureus, E. coli, Bacillus spp. and Streptococcus.spp. to different antibiotics.

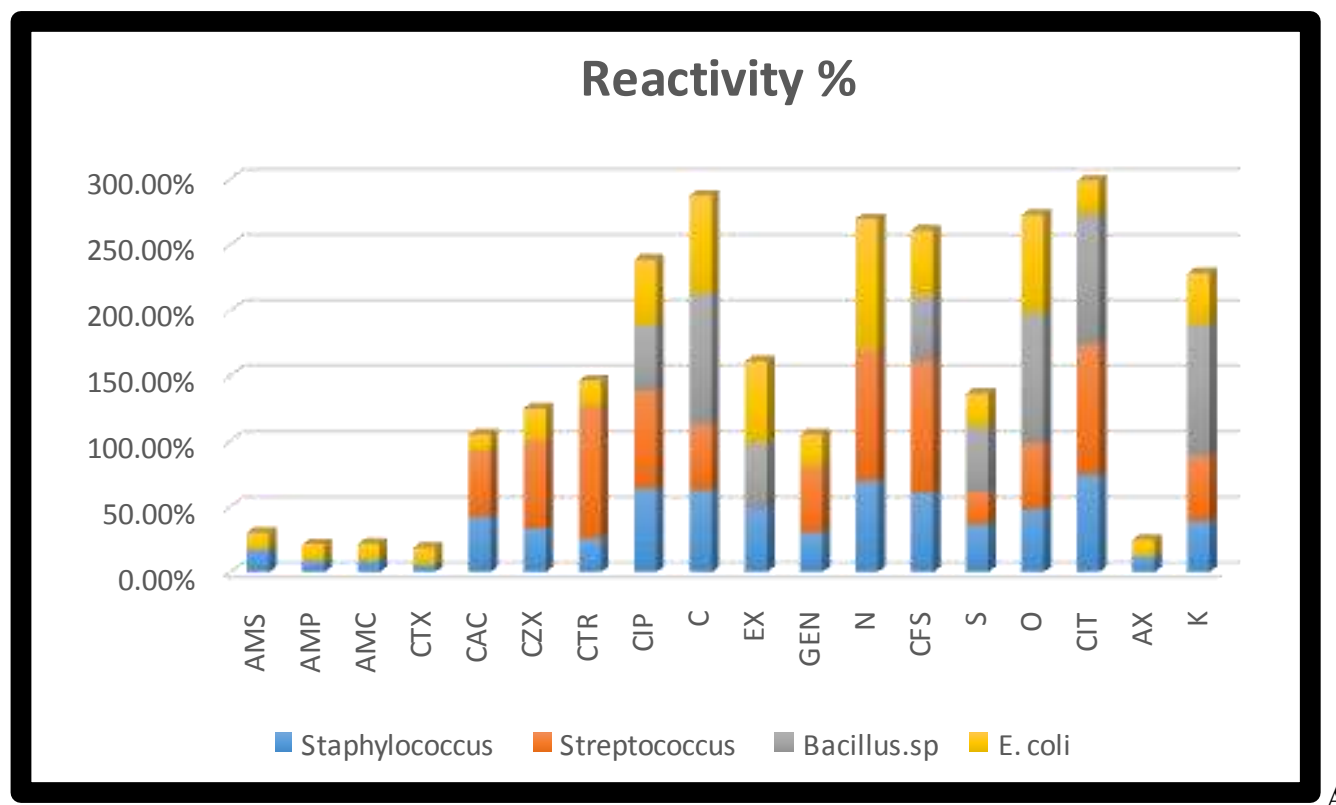

Amoxicillin/sulbactam - (AMS), Ampicillin - (AMP), Amoxyclav - (AMC), Cefotaxime - (CTX), Ceftazidime - (CAC), Ceftizoxime - (CZX), Ceftriaxone - (CTR), Ciprofloxacin - (CIP), Chloramphenicol (C), Enrofloxacin (EX), Gentamicin (GEN), Neomycin (N), Cefoperozone/ sulbactam - (CFS), Streptomycin - (S), Oxytetracycline - (O), Ceftriaxone tazobactam - (CIT), Ampicillin/Cloxacillin - (AX), Kanamycin - (K).

Fig.5b Sensitivity of Enterococcus spp., Enterobacter spp., Pseudomonas aeruginosa, Diplococci spp. and mixed infection to different antibiotics

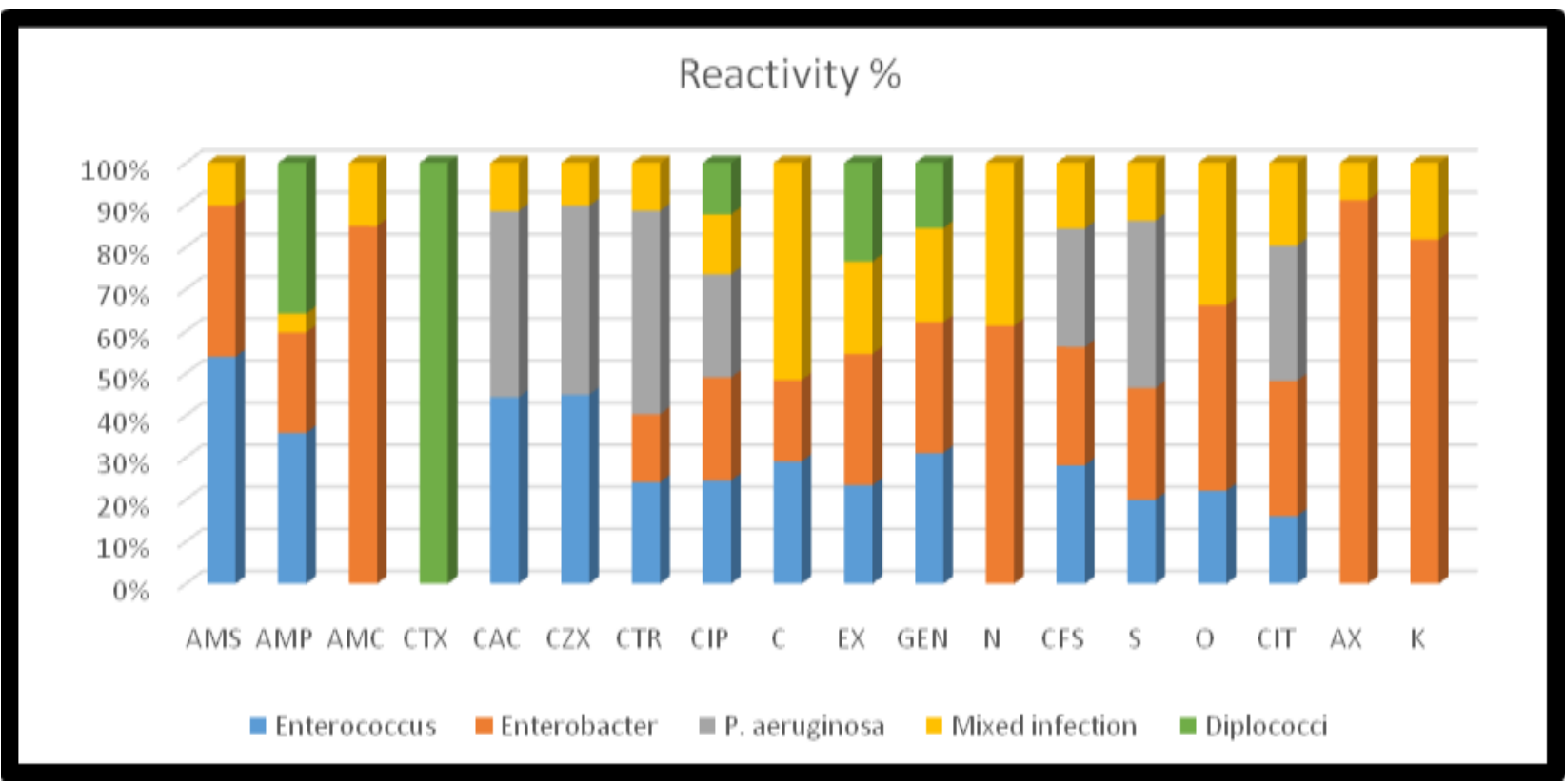

Abbreviate: Amoxicillin/sulbactam - (AMS), Ampicillin - (AMP), Amoxyclav - (AMC), Cefotaxime - (CTX), Ceftazidime - (CAC), Ceftizoxime - (CZX), Ceftriaxone - (CTR), Ciprofloxacin - (CIP), Chloramphenicol (C), Enrofloxacin (EX), Gentamicin (GEN), Neomycin (N), Cefoperozone/ sulbactam - (CFS), Streptomycin - (S), Oxytetracycline - (O), Ceftriaxone tazobactam - (CIT), Ampicillin/Cloxacillin - (AX), Kanamycin - (K) 
Overall, it has been found that Ceftriaxone/ tazobactam, Ciprofloxacin, Cefoperozone/ sulbactam, Oxytetracycline, Neomycin, Chloramphenicol are more sensitive than rest of the antibiotics.

Improper, incomplete and unscientific use of antibiotic has led to the development of resistant bacteria. General awareness among dairy farmers about hygiene, proper milking procedure is need of hour. Proper clinical investigation of cases and appropriate antibiotic and non-antibiotic based polyherbal medication will definitely help in alleviating antimicrobial resistance menace.

\section{Acknowledgements}

The authors are thankful to Mr. Mohan Ji Saxena, Managing Trustee, Ayurvet Research Foundation, for providing guidance and facilities to conduct this research work.

\section{References}

Barrow, G.I. and Feltham, R.K.A. (1993). Cowan and Steel's manual for the identification of medical bacteria. 3rd edn. Cambridge University Press, Cambridge. pp 140-143

Bauer, A.W., W.M.M. Kirby, J.S. Sherris and M. Turck (1966). Antibiotic susceptibility testing by a standard single disc method. Am. J. Clin. Pathol., 45: 493-496.

Dharamsheela Thakur, Rahul Singh, Amit
Kumar, Vikash Kumar Jha and Prabhash Kumar Singh (2018). Isolation and identification of major pathogen from clinical and subclinical mastitis milk sample and their antibiotic sensitivity evaluation. International Journal of Current Microbiology and Applied Sciences. 7: 4315-4321.

Krishnakumar S., Vikas Yadav, Anurag borthakur, K. Ravikanth (2017). Efficacy evaluation of topical herbal spray in sub clinical mastitis in bovines. World Journal of Pharmaceutical and Medical Research (WJPMR), 3(8), 172-175

Rakesh Kumar, Yudhbir Singh Rana and Anshu Sharma (2018). Comparative study of different tests for diagnosis of sub clinical mastitis in buffaloes. International Journal of Current Microbiology and Applied Sciences 7(10):520-528.

Rathi,C.L, RISBUD, S.P, Manohar, B.M (2008), Novel compositions for prevention and treatment of mastitis and metritis, WO 2008/035370 A2, March 2008

Singh, A., D. Chhabra, R. Sikrodia, S. Shukla, R. Sharda and S. Audarya (2018). Isolation of $\mathrm{E}$. coli from bovine mastitis and their antibiotic sensitivity pattern. International Journal of Current Microbiology and Applied Sciences, 7(10): 11-18 12.

\section{How to cite this article:}

Samanwita Banerjee, Anil Kanaujia, Rajkumar Yadav and Mohan JI Saxena. 2019. Mastitis Prevalence in Villages of District Sonipat and Panipat, Haryana. Int.J.Curr.Microbiol.App.Sci. 8(10): 1347-1354. doi: https://doi.org/10.20546/ijcmas.2019.810.157 